\title{
THE INFRARED ARRAY CAMERA COMPONENT OF THE SPITZER SPACE TELESCOPE EXTRAGALACTIC FIRST LOOK SURVEY
}

\author{
M. Lacy, ${ }^{1}$ G. Wilson, ${ }^{1}$ F. Masci, ${ }^{1}$ L. J. Storrie-Lombardi, ${ }^{1}$ P. N. Appleton, ${ }^{1}$ L. Armus, ${ }^{1}$ S. C. Chapman, ${ }^{1}$ \\ P. I. Choi, ${ }^{1}$ D. Fadda, ${ }^{1}$ F. Fang, ${ }^{1}$ D. T. Frayer, ${ }^{1}$ I. Heinrichsen, ${ }^{1}$ G. Helou, ${ }^{1}$ M. Im, ${ }^{2}$ S. Laine, ${ }^{1}$ \\ F. R. Marleau, ${ }^{1}$ D. L. Shupe, ${ }^{1}$ B. T. Soifer, ${ }^{1}$ G. K. Squires, ${ }^{1}$ \\ J. Surace, ${ }^{1}$ H. I. Teplitz, ${ }^{1}$ and L. Yan ${ }^{1}$ \\ Received 2004 December 30; accepted 2005 June 8
}

\begin{abstract}
We present Infrared Array Camera (IRAC) data and source catalogs from the Spitzer Space Telescope Extragalactic First Look Survey. The data were taken in four broad bands centered at nominal wavelengths of 3.6, 4.5, 5.8 , and $8.0 \mu \mathrm{m}$. A set of mosaics and catalogs have been produced that are $\approx 80 \%$ complete and $\approx 99 \%$ reliable to their chosen flux density limits. The main field survey covers $3.8 \mathrm{deg}^{2}$ and has flux density limits of 20, 25, 100, and $100 \mu \mathrm{Jy}$ at wavelengths of $3.6,4.5,5.8$, and $8.0 \mu \mathrm{m}$, respectively. The deeper "verification" survey covers $0.25 \mathrm{deg}^{2}$ with limits of $10,10,30$, and $30 \mu \mathrm{Jy}$, respectively. We also include deep data in the ELAIS-N1 field, which covers $0.041 \mathrm{deg}^{2}$ with limits of $4,3,10$, and $10 \mu \mathrm{Jy}$, respectively, but with only two wavelength coverages at a given sky position. The final bandmerged catalogs contain 103,193 objects in the main field, 12,224 in the verification field, and 5239 in ELAIS-N1. Flux densities of high signal-to-noise objects are accurate to about 10\%, and the residual systematic error in the absolute flux density scale is $\sim 2 \%-3 \%$. We have successfully extracted sources at source densities as high as 100,000 $\mathrm{deg}^{-2}$ in our deepest 3.6 and $4.5 \mu \mathrm{m}$ data. The mosaics and source catalogs will be made available through the Spitzer Science Center archive and the Infrared Science Archive.
\end{abstract}

Subject headings: catalogs — infrared: galaxies — surveys

\section{INTRODUCTION}

The extragalactic portion of the Spitzer Space Telescope (Werner et al. 2004) First Look Survey (hereafter XFLS) was one of the first observations made with Spitzer after the completion of Science Verification at the end of 2003 November. The aim of this $67 \mathrm{hr}$ survey was to characterize the extragalactic source populations observed with Spitzer down to sub-millijansky levels in the mid-infrared. ${ }^{3}$ Observations covering the survey areas were made with both the Infrared Array Camera (IRAC; Fazio et al. 2004a) and the Multiband Imaging Photometer for Spitzer (MIPS). This paper discusses the IRAC observations, the MIPS observations will be discussed in future papers (D. Fadda et al. 2005, in preparation; Frayer et al. (2005). IRAC takes images in four broad bands (termed channels). Channel 1 is centered at a nominal wavelength of $3.6 \mu \mathrm{m}$, channel 2 at $4.5 \mu \mathrm{m}$, channel 3 at $5.8 \mu \mathrm{m}$, and channel 4 at $8.0 \mu \mathrm{m} .{ }^{4}$ Light enters the instrument through two apertures: one for channels 1 and 3, and one for channels 2 and 4 . The centers of the two apertures are separated by about 6.' 5 on the sky. Beam splitters split the short- and longwavelength light in each aperture. The light in channels 1 and 2 is detected by two $256 \times 256 \mathrm{InSb}$ arrays, and that in channels 3 and 4 is detected by two $256 \times 256 \mathrm{SiAs}$ arrays. All arrays have the same pixel size, corresponding to 1 ".22 per pixel, giving a 5 ' $2 \times 5$ ' 2 field of view. The pipeline-processed IRAC data from the XFLS were publicly released at the end of 2004 April. In this paper, we describe the data analysis that we performed to

\footnotetext{
${ }^{1}$ Spitzer Science Center, Caltech, Mail Code 220-6, Pasadena, CA 91125; mlacy@ipac.caltech.edu.

2 Astronomy Program, School of Earth and Environmental Sciences, Seoul National University, Shillim-dong, Kwanak-gu, Seoul, S. Korea 2-880-9010.

3 See the First Look Survey Web site at http://ssc.spitzer.caltech.edu/fls/.

4 The actual central wavelengths are slightly different and depend on the source spectrum; see Fazio et al. (2004a) for details.
}

improve the data quality beyond the scope of the standard pipeline processing, including a discussion of the removal of artifacts from the data. We present IRAC source catalogs for the XFLS and discuss good observing practices for future surveys of this nature.

\section{DATA COLLECTION}

Nine astronomical observation requests (AORs) cover the main field survey area of $3.8 \mathrm{deg}^{2}$ centered on R.A. (J2000.0) $17^{\mathrm{h}} 18^{\mathrm{m}} 00^{\mathrm{s}}$, decl. $(\mathrm{J} 2000.0)+59^{\circ} 30^{\prime} 00^{\prime \prime}$ in a $3 \times 3$ grid in array (row, column) coordinates. Each AOR was an approximately $8 \times 8$ map with $277^{\prime \prime}$ offsets. The small five-point Gaussian dither pattern with $12 \mathrm{~s}$ frame time was used. This pattern has a mean offset of $28^{\prime \prime}$. Data in the verification area [a $0.25 \mathrm{deg}^{2}$ area within the main field centered on R.A. (J2000.0) $17^{\mathrm{h}} 17^{\mathrm{m}} 00^{\mathrm{s}}$, decl. $\left.(\mathrm{J} 2000.0)+59^{\circ} 45^{\prime} 00^{\prime \prime}\right]$ was taken using three AORs with $12 \mathrm{~s}$ frame times with the same dither and mapping strategy as the main field and three much deeper AORs with $30 \mathrm{~s}$ frame times using the first 16 points from the small cycling dither pattern, which has a mean offset of $13^{\prime \prime}$. These AORs are all contained in Spitzer program identification number (PID) 26 (PI: T. Soifer).

In addition, a second field in the ELAIS-N1 region, centered on R.A. (J2000.0) $16^{\mathrm{h}} 09^{\mathrm{m}} 20^{\mathrm{s}}$, decl. (J2000.0) +54 $57^{\prime} 00^{\prime \prime}$ was observed as part of a study of source confusion as a test field for the Great Observatories Origins Deep Survey (GOODS), and this has also been included in the XFLS (PID 196; PI: M. Dickinson). These data consisted of a two-position map separated by $312^{\prime \prime}$, with the first 36 dithers from the medium cycling pattern and a $200 \mathrm{~s}$ frame time. The map was oriented to avoid overlap between the channel 1/3 and channel 2/4 fields of view, thus, unlike the main and verification fields, a typical sky position in the image only has two channel coverage.

Details of the XFLS AORs, along with their AOR identification numbers (AORIDs) are given in Table 1. The AORs can be 
TABLE 1

Spitzer Observations of the XFLS Fields

\begin{tabular}{|c|c|c|c|c|c|c|}
\hline AORID & $\begin{array}{c}\text { Observation Date } \\
\text { (UT) }\end{array}$ & Field & $\begin{array}{c}\text { Frame Time } \\
\text { (s) }\end{array}$ & $\begin{array}{l}\text { Map Offset } \\
\quad(\operatorname{arcsec})\end{array}$ & Mapping Strategy ${ }^{\mathrm{b}}$ & Dither Pattern $^{c}$ \\
\hline $3861504(0003861504) \ldots \ldots$. & 2003 Dec 1 & Main & 12 & $-2273.5,-2550.5$ & $7 \times 9, \quad\left(277^{\prime \prime}, 277^{\prime \prime}\right)$ & 5-point Gaussian, small \\
\hline $3861760(0003861760) \ldots \ldots$. & 2003 Dec 1 & Main & 12 & $-2412.0,-138.5$ & $8 \times 9,\left(277^{\prime \prime}, 277^{\prime \prime}\right)$ & 5-point Gaussian, small \\
\hline $3862016(0003862016) \ldots \ldots .$. & 2003 Dec 2 & Main & 12 & $-2412.0,2135.0$ & $8 \times 8,\left(277^{\prime \prime}, 277^{\prime \prime}\right)$ & 5-point Gaussian, small \\
\hline $3862272(0003862272) \ldots \ldots$. & 2003 Dec 2 & Main & 12 & $-138.5,-2412.0$ & $9 \times 8,\left(277^{\prime \prime}, 277^{\prime \prime}\right)$ & 5-point Gaussian, small \\
\hline $3862528(0003862528) \ldots \ldots$. & 2003 Dec 3 & Main & 12 & $-138.5,-138.5$ & $9 \times 9,\left(277^{\prime \prime}, 277^{\prime \prime}\right)$ & 5-point Gaussian, small \\
\hline $3862784(0003862784) \ldots \ldots$. & 2003 Dec 3 & Main & 12 & $-138.5,2273.5$ & $9 \times 9,\left(277^{\prime \prime}, 277^{\prime \prime}\right)$ & 5-point Gaussian, small \\
\hline $3863040(0003863040) \ldots \ldots$. & 2003 Dec 3 & Main & 12 & $2273.5,-2273.5$ & $9 \times 7,\left(277^{\prime \prime}, 277^{\prime \prime}\right)$ & 5-point Gaussian, small \\
\hline $38633296(0003863296) \ldots .$. & 2003 Dec 4 & Main & 12 & $2273.5,-138.5$ & $9 \times 9,\left(277^{\prime \prime}, 277^{\prime \prime}\right)$ & 5-point Gaussian, small \\
\hline $3863552(0003863552) \ldots \ldots$. & 2003 Dec 4 & Main & 12 & $2135.0,2412.0$ & $8 \times 10,\left(277^{\prime \prime}, 277^{\prime \prime}\right)$ & 5-point Gaussian, small \\
\hline $3866880(0003866880) \ldots \ldots .$. & 2003 Dec 5 & Verification & 12 & $0.0,0.0$ & $4 \times 7,\left(277^{\prime \prime}, 277^{\prime \prime}\right)$ & 5-point Gaussian, small \\
\hline $7676928(0007676928) \ldots \ldots$. & 2003 Dec 5 & Verification & 12 & $-830.0,-150.0$ & $2 \times 6,\left(277^{\prime \prime}, 277^{\prime \prime}\right)$ & 5-point Gaussian, small \\
\hline $7677184(0007677184) \ldots \ldots$. & 2003 Dec 5 & Verification & 12 & $830.0,150.0$ & $2 \times 6,\left(277^{\prime \prime}, 277^{\prime \prime}\right)$ & 5-point Gaussian, small \\
\hline $3867136(0003867136) \ldots \ldots .$. & 2003 Dec 5 & Verification & 30 & 0,0 & $4 \times 5,\left(277^{\prime \prime}, 277^{\prime \prime}\right)$ & Points $1-16$ from small cycling \\
\hline $3867392(0003867392) \ldots \ldots .$. & 2003 Dec 5 & Verification & 30 & $830.0,150.0$ & $2 \times 5,\left(277^{\prime \prime}, 277^{\prime \prime}\right)$ & Points $1-16$ from small cycling \\
\hline $3867648(0003867648) \ldots \ldots$. & 2003 Dec 6 & Verification & 30 & $-830.0,-150.0$ & $2 \times 5,\left(277^{\prime \prime}, 277^{\prime \prime}\right)$ & Points $1-16$ from small cycling \\
\hline $6006016(0003867648) \ldots \ldots$. & 2003 Dec 28 & ELAIS-N1 & 200 & $201.65,0.0$ & $1 \times 2,\left(292^{\prime \prime}, 312^{\prime \prime}\right)$ & Points $1-36$ from medium cycling \\
\hline
\end{tabular}

Note.-Links to data sets in this table are available in the electronic edition of the Supplement.

${ }^{a}$ Relative to the appropriate field center, in array coordinates.

b Size of map (row $\times$ column), mapping steps in array coordinates.

c These are standard IRAC dither patterns, described in the Spitzer Observers' Manual.

downloaded using the Spot tool, ${ }^{5}$ selecting the "View Program" option, and specifying the appropriate PID.

\section{DATA ANALYSIS}

\subsection{Pipeline Processing}

The main and verification field data were run through the S10.5 version of the Spitzer Science Center (SSC) pipeline, described in the IRAC Data Handbook. ${ }^{6}$ Those for the ELAIS-N1 field used the S9.5 version of the pipeline. Between S9.5 and S10.5 the treatment of the darks was improved, but the long frames in the ELAIS-N1 data are background dominated, so the improvement between S9.5 and S10.5 was negligible for these data. For each IRAC frame (termed a data collection event, or DCE) the IRAC pipeline produces processed images, the basic calibrated data (BCD), and corresponding masks (the DCE masks, or Dmasks) that we used as the starting point for our analysis. The masks flag potential problems with the data such as saturated pixels, strong radiation hits, or corrupted $/ \mathrm{missing}$ data.

\subsection{Postpipeline Processing}

The IRAC data contain a number of artifacts that are not yet removed well by the SSC pipeline. Some of these were removed in postpipeline processing steps.

Bright stars and cosmic rays systematically decrease the bias level in the columns in which they lie in channels 1 and 2 (Fig. 1). This "column pulldown" was removed using an algorithm developed and implemented by L. Moustakas \& D. Stern (2004, private communication). This masks objects in the frame to produce a background image, then searches for discrepant columns, correcting them by adding a constant to bring the mean background value in the column up to that of its neighbors. Multiplexer bleed ("muxbleed") in channels 1 and 2 from stars and cosmic rays with peak fluxes in the linear regime was partly

\footnotetext{
${ }^{5}$ See http://ssc.spitzer.caltech.edu/propkit/spot/.

${ }^{6}$ Available from the SSC Web site (http://ssc.spitzer.caltech.edu/irac/dh/).
}

corrected by the SSC pipeline, but that from the brightest, saturated stars was not. To correct these, we searched for bright stars in the frame, then split the data into the four amplifier outputs (every fourth column of data passes through the same output amplifier). For each of the four outputs, we fitted the rows within 5 pixels of the row containing the star with a straight line using columns outside \pm 30 pixels of the star, subtracted the fit, and reassembled the frame. In channels 3 and 4, banding effects (predominately optical in origin, produced by scattering of light within the detector) were present (Fig. 2). These resulted in the rows and columns containing the bright stars to be elevated above the background. The banding artifacts were removed in a similar fashion to the pulldown and muxbleed in channels 1 and 2 , though in this case the outputs were not split. Muxbleed from extremely bright stars in channels 1 and 2 can result in a large fraction of the array being offset from the mean level. This was corrected by searching for saturated stars, then averaging the rows above and below the star in each of the four outputs, fitting a line to the mean of each row and subtracting the fit. Further details on and examples of these artifacts may be found in the IRAC Data Handbook.

The IRAC data have a number of problems that affect the background level across frames. The "first frame effect"the variation in the dark that depends on the length of time since the previous exposure - is partly corrected in the pipeline, but some residual variation in the dark remains, particularly in channel 3. There are two reasons for this. First, the first frame calibrations (taken prior to launch), did not cover a sufficiently large time range to calibrate the longest-term variations, and second, they were taken prior to the decision to anneal the IRAC arrays regularly to remove long-term latents. To remove the dark variations to first order, a plane was fitted to produce a flat image with a constant background. This dealt with much of the uncorrected first frame effect. However, background variations remained from three sources that produce background variations on scales smaller than the array. These were residual first-frame/ array relaxation effects, long-term latents (particularly noticeable in channel 1), and some real positional variations in the 


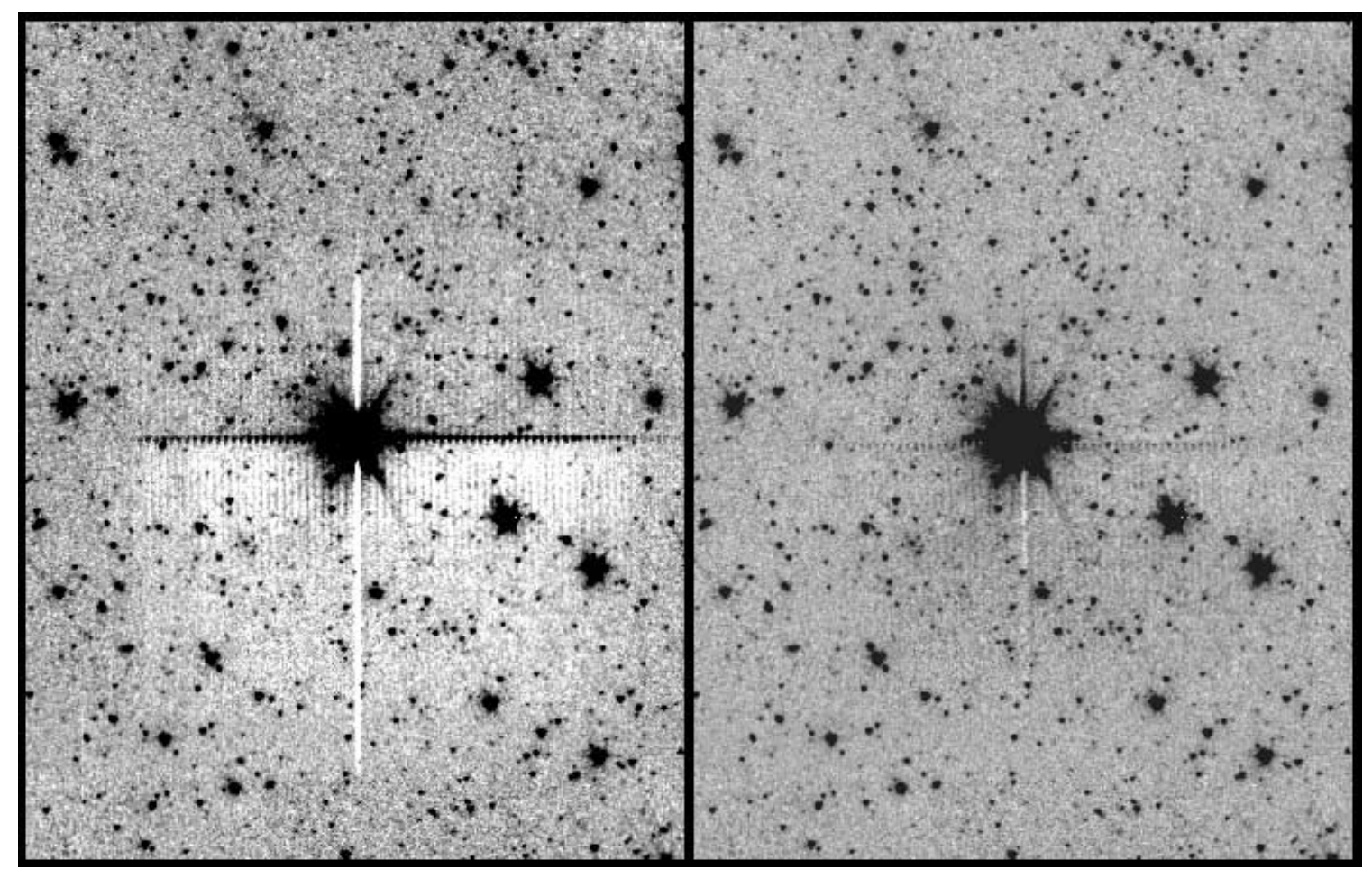

FIg. 1.-Bright star in the channel 1 mosaic shown before (left) and after (right) the postpipeline processing was applied. The star shows all the artifacts produced by a bright star in channels 1 and 2: column pulldown, the reduction in the data values in columns directly above and below the star, muxbleed (raising the level of pixels in rows either side of the star), and the offset in the background level produced by extremely strong muxbleed affecting all columns below the star and also producing striations in the background level. The postpipeline processing is able to remove most of the artifacts.

background level due to high Galactic latitude dust emission in channel 4. We therefore subtracted a "delta dark" from each frame. In the data taken with the $12 \mathrm{~s}$ frame times, channels 1,2 , and 4 were adequately corrected (in the sense that the variations in the background level were reduced to be similar in magnitude to the noise level) by subtracting a $\pm 1.5 \sigma$ clipped median of the whole pixel stack from all the frames. Channel 3 required a running median of 15 of the $\pm 1.5 \sigma$ clipped pixel stack to produce acceptable results. The $30 \mathrm{~s}$ frame time data were more problematic. Channel 1 proved to be particularly challenging. The

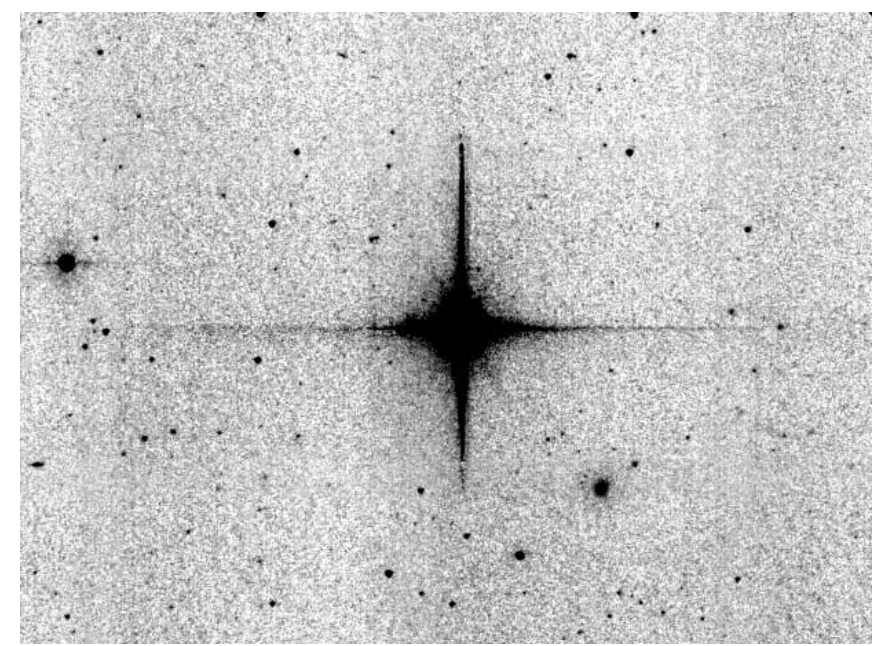

small dither pattern proved too small to effectively remove latent images from bright stars. In addition, the longer frame times led to a gradual build-up of latents over the whole array. A running median of 24 of the $\pm 1.5 \sigma$ clipped pixel stack produced good results for all but the last two pointings of the AORs (which happened to contain bright stars). These frames had a 48-frame running median subtracted instead. In channel 3 the first $30 \mathrm{~s}$ frame of the AOR was essentially uncorrectable and was excluded from the mosaics. For the rest, a 36-frame running median of the $\pm 1.5 \sigma$ clipped pixel stack was found to be adequate. A straight

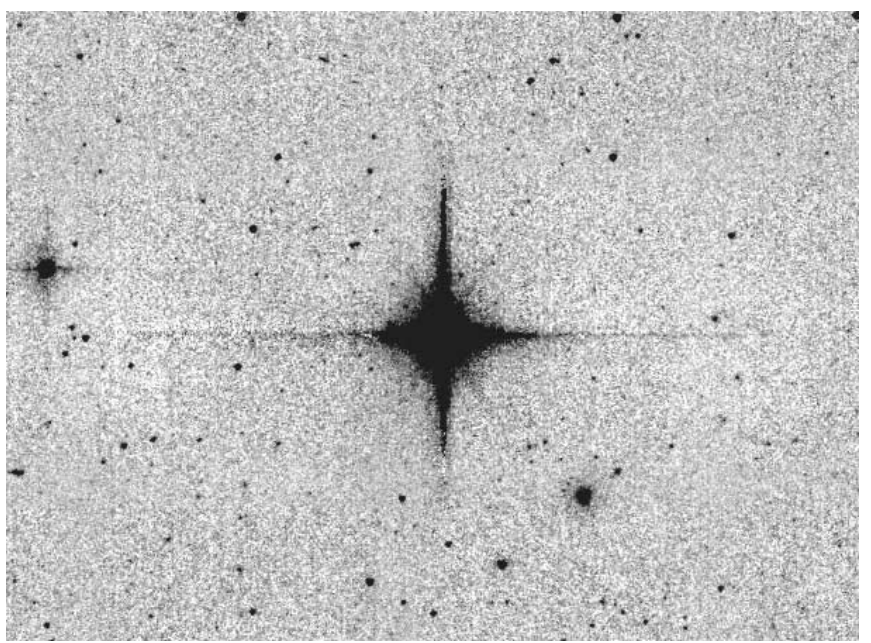

FIG. 2.-Bright star in the channel 3 mosaic shown before (left) and after (right) the postpipeline processing was applied. The star shows the combination of optical

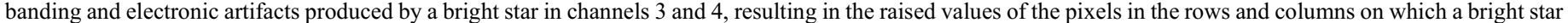

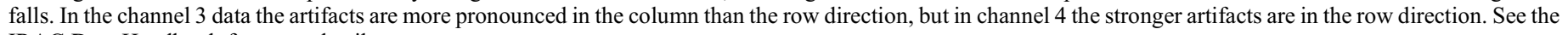
IRAC Data Handbook for more details. 
TABLE 2

Values of the More Important Mosaicker and SExtractor Parameters

\begin{tabular}{|c|c|c|c|c|c|}
\hline $\begin{array}{l}\text { Program } \\
\text { (Module) }\end{array}$ & Parameter & Channel 1 & Channel 2 & Channel 3 & Channel 4 \\
\hline \multicolumn{6}{|c|}{ Common to All } \\
\hline Mopex (DETECT) .... & Detection_Max_Area & 9 & 9 & 9 & 9 \\
\hline Mopex (DETECT) & Detection_Min_Area & 0 & 0 & 0 & 0 \\
\hline 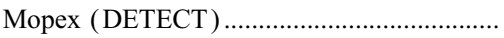 & Detection_Threshold & 5 & 5 & 5 & 5 \\
\hline Mopex (MOSAICINT) .................................... & INTERP_METHOD & 1 & 1 & 1 & 1 \\
\hline Mopex (MOSAICDUALOUTLIER) ............. & MIN_OUTL_IMAGE & 2 & 2 & 2 & 2 \\
\hline Mopex (MOSAICDUALOUTLIER) ............. & MIN_OUTL_FRAC & 0.2 & 0.2 & 0.2 & 0.2 \\
\hline Mopex (MOSAICOUTLIER) .......................... & THRESH_OPTION & 2 & 2 & 2 & 2 \\
\hline Mopex (MOSAICOUTLIER) ........................... & BOTTOM_THRESHOLD & 4 & 4 & 4 & 4 \\
\hline Mopex (MOSAICOUTLIER) .......................... & TOP_THRESHOLD & 4 & 4 & 4 & 4 \\
\hline Mopex (MOSAICOUTLIER) .......................... & MIN_PIX_NUM & 3 & 3 & 3 & 3 \\
\hline Mopex (MOSAICRMASK) .............................. & MIN_COVERAGE & 3 & 3 & 3 & 3 \\
\hline Mopex (MOSAICRMASK) ............................. & MAX_COVERAGE & 100 & 100 & 100 & 100 \\
\hline 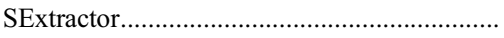 & DETECT_MINAREA & 3 & 3 & 3 & 3 \\
\hline SExtractor........... & DETECT_THRESH & 2 & 2 & 2 & 2 \\
\hline SExtractor......... & ANALYSIS_THRESH & 2 & 2 & 2 & 2 \\
\hline SExtractor......... & FILTER & $\mathrm{N}$ & $\mathrm{N}$ & $\mathrm{N}$ & $\mathrm{N}$ \\
\hline . & DEBLEND_NTHRESH & 32 & 32 & 32 & 32 \\
\hline SExtractor.......... & DEBLEND_MINCONT & 0.0001 & 0.0001 & 0.0001 & 0.0001 \\
\hline SExtractor.......... & BACK_SIZE & 16 & 16 & 16 & 16 \\
\hline 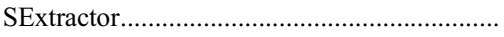 & BACK_FILTERSIZE & 3 & 3 & 3 & 3 \\
\hline SExtractor........... & BACKPHOTO_TYPE & LOCAL & LOCAL & LOCAL & LOCAL \\
\hline \multicolumn{6}{|c|}{ Main Field } \\
\hline Mopex .......... & MOSAIC_PIXEL_RATIO_X & 1 & 1 & 1 & 1 \\
\hline Mopex .............. & MOSAIC_PIXEL_RATIO_Y & 1 & 1 & 1 & 1 \\
\hline Mopex (MOSAICRMASK) ............................. & RM_THRESH & 0.8 & 0.8 & 0.05 & 0.05 \\
\hline SExtractor & SATUR_LEVEL & 325 & 463 & 2274 & 878 \\
\hline 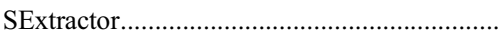 & GAIN & 309 & 280 & 67 & 195 \\
\hline \multicolumn{6}{|c|}{ Verification Field } \\
\hline Mopex ...... & MOSAIC_PIXEL_RATIO_X & 1 & 1 & 1 & 1 \\
\hline 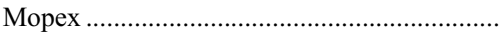 & MOSAIC_PIXEL_RATIO_Y & 1 & 1 & 1 & 1 \\
\hline Mopex (MOSAICRMASK) .............................. & RM_THRESH & 0.8 & 0.8 & 0.05 & 0.05 \\
\hline 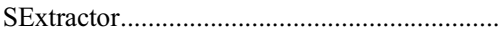 & SATUR_LEVEL & 126 & 180 & 883 & 341 \\
\hline SExtractor & GAIN & 786 & 721 & 67 & 172 \\
\hline \multicolumn{6}{|c|}{ ELAIS-N1 Field } \\
\hline 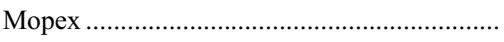 & MOSAIC_PIXEL_RATIO_X & 2 & 2 & 2 & 2 \\
\hline 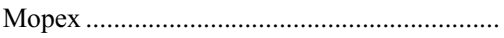 & MOSAIC_PIXEL_RATIO_Y & 2 & 2 & 2 & 2 \\
\hline Mopex (MOSAICRMASK) .............................. & RM_THRESH & 0.8 & 0.8 & 0.8 & 0.8 \\
\hline 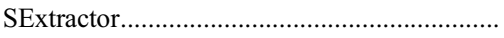 & SATUR_LEVEL & 17.4 & 24.9 & 122 & 195 \\
\hline 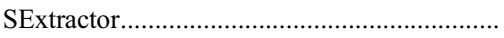 & GAIN & 5679 & 5210 & 1244 & 876 \\
\hline
\end{tabular}

median of the clipped frames, as used for the $12 \mathrm{~s}$ data, was found to be adequate in channels 2 and 4 .

To mask scattered light from sources off the detector array, ${ }^{7}$ a stray light masking program developed by R. Arendt and ML was used to place masks into the Dmask file. This program uses a list of bright stars from the Two Micron All Sky Survey (2MASS; Cutri et al. 2003) to predict the positions of light scattered from the focal plane assembly (FPA) cover (the principal source of scattered light in channels 1 and 2), and scattering off the edge of the detector (the principal source of scattering in channels 3 and 4). These masks have been applied to the final mosaics.

\footnotetext{
${ }^{7}$ For details of scattered light in IRAC see the Spitzer Observers' Manual http://ssc.spitzer.caltech.edu/documents/som/.
}

Software for IRAC artifact correction and masking, including up to date versions of much of the code described above may be found on the Spitzer contributed software Web site. ${ }^{8}$

The SSC pointing refinement module (Masci et al. 2004) was run on all the channel 1 and channel 2 BCDs. This module improves the pointing of the BCDs by both registering each BCD with respect to overlapping BCDs ("relative refinement") and by registering the result with stars from the 2MASS cata$\log$ ("absolute refinement"). To apply these corrections to the channel 3 and 4 BCDs, which have too few stars for a reliable absolute correction, we computed a weighted average of the channel 1 and channel 2 correction offsets, applied these new

\footnotetext{
${ }^{8}$ See http://ssc.spitzer.caltech.edu/archanaly/contributed/browse.html.
} 
offsets to all channel 1,2,3, and 4 BCDs, and finally computed the refined pointing for all the BCDs. The mosaics constructed using the refined pointings from the $\mathrm{BCD}$ are well registered to the 2MASS frame (see $\S 5$ below).

\subsection{Production of the Mosaics}

The SSC mosaicking software Mopex ${ }^{9}$ was used to mosaic the individual BCD images. Mopex consists of a set of modules combined in a PERL wrapper script. Which modules are used, and the parameters that control the modules, are determined by namelist files. The namelists used were similar to the one given in the IRAC Data Handbook. Standard linear interpolation was used for all the mosaics. Two modes of outlier rejection were employed, the "dual outlier" rejection, which uses a combination of spatial and temporal criteria to identify outliers, and a purely temporal ("multiframe") technique, which uses the pixel stack at a given image position to identify outliers. The multiframe technique is the most effective when the coverage is high $(\gtrsim 4)$, but the dual outlier method is better on the lower coverage regions on the edge of the mosaics, or in regions masked by the Dmasks. The single frame outlier module, which relies purely on a spatial criterion to determine outliers, was not used. The diffuse cosmic rays in channels 3 and 4 required that the masks be grown around detected outlier pixels in the lower coverage data. This was achieved in practice by setting the parameter RMTHRESH to a low value (0.05) in the mosaicker channel 3 and 4 namelists for the main and verification fields. Coverage maps for each mosaic were output by the mosaicker and were checked by eye for evidence of overzealous outlier rejection. To help with deblending sources, the ELAIS-N1 data were mosaicked with a $2: 1$ pixel ratio to better sample the data. The main field and verification strip retained their original sampling. The Mopex namelists used for the XFLS will be made available on the XFLS Web site so others can reproduce our results should they wish to do so. Some of the more important parameter choices are listed in Table 2 .

\section{PRODUCTION OF THE SOURCE CATALOGS}

Single-band catalogs were produced from each mosaic image using SExtractor (Bertin \& Arnouts 1996). The coverage maps produced by Mopex were used as weight images. We have optimized the photometry for the faintest objects. A background mesh size of 16 pixels with a filter width of three was used, and the "local" background (measured in a 24 pixel thick annulus) applied. Large, extended objects are thus likely to have incorrect fluxes in the catalog and should have their fluxes remeasured from the mosaics. Four fixed aperture fluxes (aperture

\footnotetext{
${ }^{9}$ Described in http://ssc.spitzer.caltech.edu/postbcd/doc/mosaiker.pdf.
}

TABLE 3

Aperture Corrections

\begin{tabular}{|c|c|c|c|c|}
\hline \multirow{2}{*}{$\begin{array}{l}\text { APERTURE } \\
\text { (arcsec) }\end{array}$} & \multicolumn{4}{|c|}{ Correction Applied } \\
\hline & Channel 1 & Channel 2 & Channel 3 & Channel 4 \\
\hline $6.00 \ldots \ldots \ldots \ldots \ldots \ldots \ldots$ & 1.167 & 1.213 & 1.237 & 1.466 \\
\hline $9.26 \ldots \ldots \ldots \ldots \ldots \ldots . .$. & 1.091 & 1.117 & 1.100 & 1.165 \\
\hline 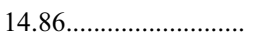 & 1.042 & 1.048 & 1.042 & 1.066 \\
\hline $24.4 \ldots \ldots \ldots \ldots \ldots \ldots \ldots$ & 1.000 & 1.000 & 1.000 & 1.000 \\
\hline
\end{tabular}

TABLE 4

IMAGE Flag VALUeS

\begin{tabular}{cll}
\hline \hline Bit & \multicolumn{1}{c}{ Meaning } & \multicolumn{1}{c}{ Set by } \\
\hline $0 \ldots \ldots$. & More than $10 \%$ area affected by bad pixels & SExtractor \\
$1 \ldots \ldots$. & Object originally blended with another one & SExtractor \\
$2 \ldots \ldots$. & At least one object pixel is saturated & SExtractor \\
$3 \ldots \ldots$. & Object is truncated at image boundary & SExtractor \\
$4 \ldots \ldots$. & Object's aperture data are incomplete or corrupted & SExtractor \\
$5 \ldots \ldots$. & Object's isophotal data are incomplete or corrupted & SExtractor \\
$6 \ldots \ldots$. & A memory overflow occurred during deblending & SExtractor \\
$7 \ldots \ldots$. & A memory overflow occurred during extraction & SExtractor \\
$8 \ldots \ldots$. & Region affected by muxbleed, pulldown or banding & XFLS software \\
$9 \ldots \ldots$. & Object in halo of bright star & XFLS software \\
$10 \ldots .$. & Object contaminated by latent image & Hand \\
\hline
\end{tabular}

diameters 6".00, 9".26, 14".86, and 24."40) plus an isophotal flux were measured. The IRAC point spread function has broad wings compared to typical ground-based data, and aperture corrections are significant (see the IRAC Data Handbook for further details). The largest aperture diameter (24".40) is the same diameter that the IRAC calibration stars are measured in. The fluxes of the calibration stars in this aperture are considered the total fluxes in the S9.5 and S10.5 pipeline processed data, so this aperture requires no correction. The smaller apertures were picked to match those used by the Sloan Digital Sky Survey (SDSS). For these, aperture corrections were applied using a lookup table derived from measurements of bright stars in the XFLS data, and checked for consistency with those derived by the IRAC instrument team and those in the IRAC Data Handbook (see Table 3). Although these corrections are not quite appropriate for the typical XFLS galaxy, which is slightly extended in IRAC images, our simulations showed that the application of this correction nevertheless significantly improved the flux densities. One of the aperture fluxes is taken as a "best" flux in the catalog. The "best" aperture was chosen by comparing the geometric mean radius of the isophote $\left[r_{m}=(A / \pi)^{1 / 2}\right.$, where $A$ is the isophotal area] to each fixed aperture diameter. This radius is compared to the radii of the four apertures $r_{1}, r_{2}, r_{3}$ and $r_{4}$. If $r_{m}<1.1 r_{1}$, then the "best" aperture radius, $r_{b}$, is set to $r_{1}$; if $1.1 r_{1}<r_{m} \leq 1.1 r_{2}$, then $r_{b}=r_{2}$, etc. If $r_{m} \geq 1.1 r_{4}$, then the isophotal flux is used as the "best" flux. Although somewhat arbitrary, this procedure ensures that an aperture appropriate to the isophotal size of the source is selected as "best," and thus reduces noise and confusion in the flux density measurement.

Flag images based on the results of the muxbleed, pulldown, and banding correctors, latent image masking in the final mosaic (applied by hand), and halos of bright stars (using sizes predicted from 2MASS $K$ magnitudes) were applied to the SExtractor catalogs. Objects whose isophotes cross nonzero pixels in the flag image have a value in the image flag field derived from ORing together the flag image pixels that fall within the object isophotes. Flag values and their meaning, for both the flag values output by SExtractor and those added by applying the flag image, are listed in Table 4.

The four IRAC single-band catalogs were also merged into a single, four-band catalog. The matching procedure began by going through each source in the band 1 catalog and searching for a match within a radius of 1.5 pixels in bands $2-4$. Sources in band 2 unmatched to band 1 sources were then matched to bands 3 and 4 , and finally sources in band 3 unmatched to bands 1 and 2 were then matched to band 4 . The radius of 1.5 pixels was chosen as a result of trying several different match radii 
TABLE 5

Morphological Breakdown for XFLS Sources Close to the Survey Limits

\begin{tabular}{|c|c|c|c|c|c|c|c|}
\hline \multirow[b]{2}{*}{ Channel } & \multirow{2}{*}{$\begin{array}{c}\text { FLUX RANGE } \\
(\mu \mathrm{Jy})\end{array}$} & \multicolumn{4}{|c|}{ FRACTION } & \multirow{2}{*}{$\begin{array}{c}\text { MEDIAN } r_{1 / 2} \\
(\operatorname{arcsec})\end{array}$} & \multirow{2}{*}{$\begin{array}{c}\text { RANGE IN } r_{1 / 2} \\
(\operatorname{arcsec})\end{array}$} \\
\hline & & Confused & Point & Disk & de Vaucouleurs & & \\
\hline 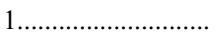 & $20-30$ & $0 / 10$ & $2 / 10$ & $6 / 10$ & $2 / 10$ & 0.4 & $0.2-0.7$ \\
\hline 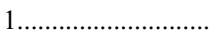 & $10-20$ & $0 / 10$ & $3 / 10$ & $7 / 10$ & $0 / 10$ & 0.4 & $0.3-1.1$ \\
\hline 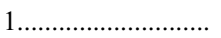 & $3-4$ & $1 / 10$ & $0 / 10$ & $8 / 10$ & $1 / 10$ & 0.07 & $0.03-0.18$ \\
\hline 4 & $100-120$ & $0 / 10$ & $6 / 10$ & $3 / 10$ & $1 / 10$ & 0.3 & $0.2-1.4$ \\
\hline 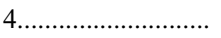 & $30-40$ & $1 / 10$ & $3 / 10$ & $5 / 10$ & $1 / 10$ & 0.4 & $0.2-0.8$ \\
\hline 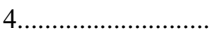 & $10-20$ & $1 / 10$ & $0 / 10$ & $7 / 10$ & $2 / 10$ & 0.25 & $0.1-0.5$ \\
\hline
\end{tabular}

between 0.5 and 2.5 pixels and examining the number of matches between each band. If the match radius is smaller than the mean position uncertainty due to random errors, the number of matches increases rapidly with increasing match radius. This rate of increase falls off when the increase in the number of matches is mostly due to chance coincidences. The optimum radius is, of course, a function of band, but the similarity of the PSFs in the IRAC bands is enough that a single radius of 1.5 pixels is a good pick for all four bands. The fluxes are "best" fluxes as described above with the aperture set to that of the shortest wavelength band in which the source is detected. The greater of the singleband catalog limit, or a $3 \sigma$ limit were placed on nondetections (or negative aperture flux densities). The detection flag field in the four-band catalog has bit 0 set for a channel 1 detection, bit 1 for channel 2, bit 2 for channel 3, and bit 3 for channel 4 .

\section{POSITIONAL ACCURACY}

For 2MASS sources in the main field detected in all four bands above the IRAC catalog flux limits (3223 sources; typical flux densities of $\approx 1 \mathrm{mJy}$ in channel 1 ), the mean radial position error with respect to 2 MASS is 0.25 , with no measurable mean offset. This degrades at faint flux levels due to both noise and source confusion. Close to the flux limits of the catalogs our simulations suggest the positional error is $\approx 1^{\prime \prime}$.

\section{COMPLETENESS}

A series of simulations were run to estimate the completeness of the main, verification, and ELAIS-N1 surveys. We define completeness as the chances of detecting a source placed at a random position in the field. Many sources are partially resolved even at the limit of the surveys. To account for this in the completeness simulations we needed to obtain an estimate of the range of source sizes near the survey limits. We used $i$-band Hubble Space Telescope images from the Advanced Camera for Surveys (ACS) taken as parallel data in program GO-9753 (PI: L. Storrie-Lombardi) for which mosaics had been produced by I. Drozdovsky (2005, private communication) to study the morphologies of objects in the main and verification fields. For ELAIS-N1 no HST data were available, but the field is sufficiently deep that IRAC and ACS data from the Great Observatories Origins Deep Survey (GOODS) in the Hubble Deep Field North (HDFN) could be used instead. Ten galaxies close to the flux limits of the channel 1 and channel 4 catalogs of each of the three surveys (main, verification, and ELAIS-N1) were fitted using software originally developed for quasar host galaxy fitting (Lacy et al. 2002). The results of this analysis are shown in Table 5. At the flux density levels of the main and verification surveys, stars contribute $\sim 30 \%$ of the sources, but these dis- appear in the ELAIS-N1 survey. The median galaxy half-light radius $\left(r_{1 / 2}\right)$ is 0 ." 4 in the main and verification surveys falling to $<0^{\prime \prime} 1$ in the channel 1 ELAIS-N1 data, and 0.25 in the channel 4 ELAIS-N1 data. The distribution of galaxy types is field-galaxy like, with $\approx 20 \%$ ellipticals, $\approx 80 \%$ spirals/irregulars.

The scale sizes of sources in the main and verification fields mean that a significant fraction of these objects will be marginally resolved by Spitzer. In channel 1, the FWHM of the PSF is 1."8, similar to the FWHM of an exponential disk galaxy with $r_{1 / 2}=0 . " 5$. In the ELAIS-N1 field, however, the scale sizes are sufficiently small that they can be treated as point sources for the purposes of completeness analysis. We therefore only modeled extended sources in the main and verification fields. For modeling the completeness in these fields, artificial sources were added to the mosaics with fluxes, sizes, and profiles that are representative of the IRAC galaxy population close to the survey limit based on the averaged results of our fitting. Specifically, we picked the model population as follows: $30 \%$ point source, $60 \% r_{1 / 2}=0$ " 4 disk galaxies and $10 \% r_{1 / 2}=0$ " 4 de Vaucouleurs galaxies. For channels 3 and 4 close to the main field flux limit, we increased the fraction of point sources to reflect the higher number of stars, using a mixture of $60 \%$ point sources, $30 \%$ $r_{1 / 2}=0 . " 4$ disk galaxies and $10 \% r_{1 / 2}=0$ ". 4 de Vaucouleurs galaxies. We then estimated the completeness by comparing the number of these artificial objects of a given flux density appearing in the SExtractor catalogs with the number known to have been added to the mosaics. The completeness was not a strong function of point versus extended source for the range of models used, or of model type.

The completeness plots for the main and verification fields are shown in Fig. 3. The ELAIS-N1 field has a very high source density in channels 1 and 2. For this field we adopted a slightly different strategy by picking a typical field source from the mosaic, scaling it by different factors, and inserting 1000 clones of this source into the mosaic. The results are shown in Figure 4.

We also tried using the verification data to check the completeness of the main field catalog by examining the fraction of verification field sources that were detected in the overlapping region of the main field catalog as a function of flux density close to the survey limits in the main field. This gave completeness values higher by $10 \%-15 \%$ close to our adopted survey limits. This result was not unexpected because a bright source will cause source confusion in both the main and the verification field, but provided a useful check on the simulations.

We would like to emphasize that our completeness estimates are only approximate, particularly for the ELAIS-N1 channel 1 and 2 data, which are close to the confusion limit. A more careful analysis of completeness in this confused regime (see, 

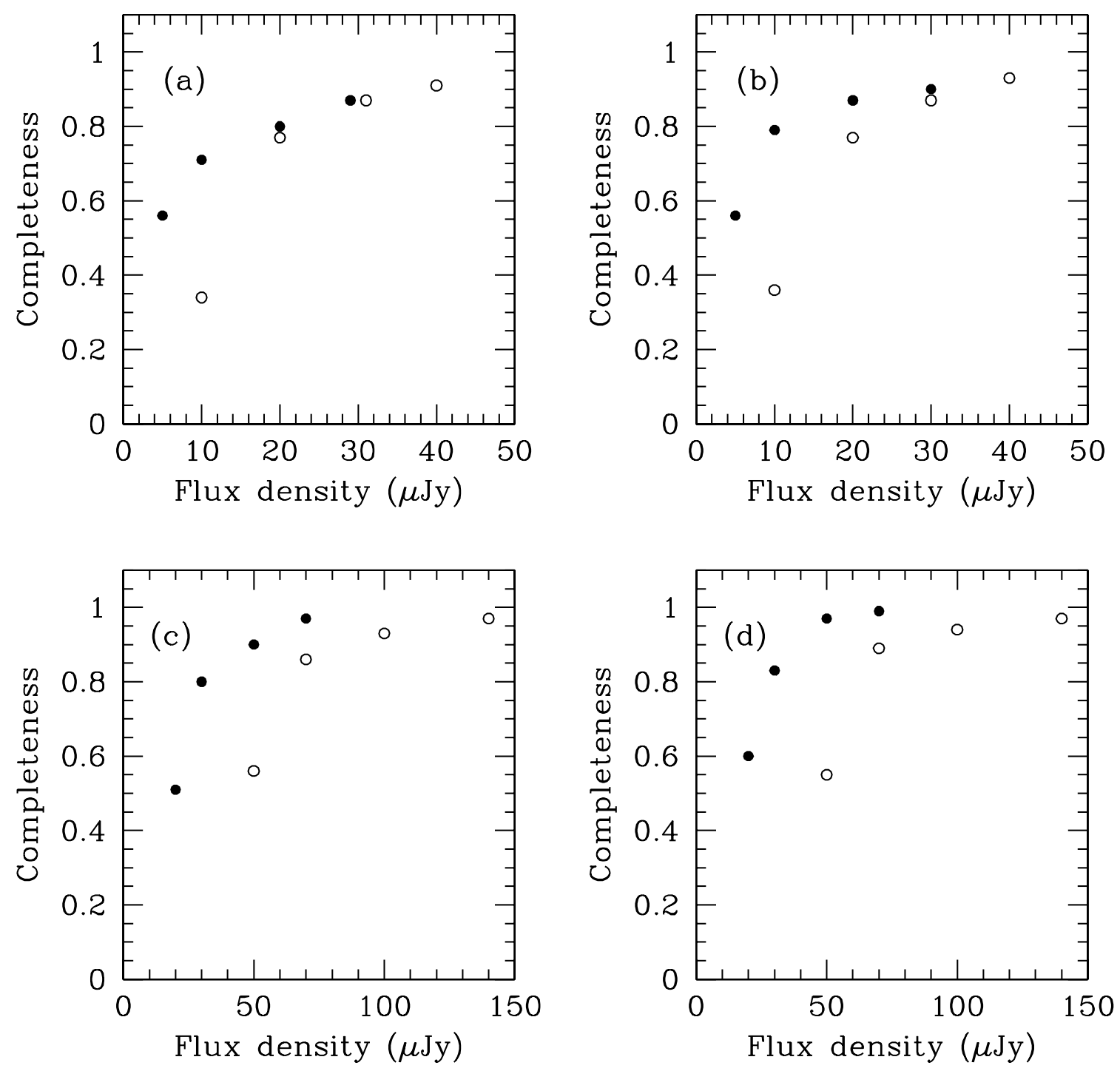

FIG. 3.-Results of completeness tests on the main and verification fields. The results for the main field are shown as open symbols, those for the verification field as closed symbols. The panels are for $(a)$ channel $1,(b)$ channel 2, (c) channel 3, and $(d)$ channel 4.

e.g., Chary et al. 2004) will be necessary for accurate estimates of source counts at the faintest flux density levels in these data.

\section{PHOTOMETRIC ACCURACY}

An estimate of the uncertainty is associated with each flux density measurement in the catalog. This includes the uncertainties output by SExtractor, which are statistical in nature, and the known sources of systematic uncertainties. These have been quantified by the IRAC Instrument and Instrument Support teams (private communications, though see discussions in the IRAC Data Handbook) and are expected to amount to $\approx 10 \%$. Most of this is due to color-dependence in the flat field and is manifest as scatter in the cataloged fluxes. This value of $10 \%$ was thus added in quadrature to the uncertainties from SExtractor. We have used three approaches to checking the systematic photometric errors. The recovery of fluxes from artificial sources was used to examine the uncertainties and biases in source measurement, in particular those associated with faint, slightly extended sources due to the small apertures and the consequent uncertainty in the aperture correction. Comparison was also made between fluxes measured for objects in the main field and the (much deeper) verification field. Finally, comparison was made to 2MASS photometry of field stars.

\subsection{Fluxes of Artificial Sources}

The catalog fluxes of the artificial sources used in the completeness tests were compared to their true fluxes. This tests the accuracy and effectiveness of our aperture corrections and photometric algorithms but does not account for the color-dependent flat-field effect.

These tests showed that, on average, more than $90 \%$ of the flux is recovered for pointlike or small sources close to the flux limit of the catalogs. Below the flux limits, the fraction of the model flux recovered falls fast. The most likely reason for this bias is source confusion and variations in the background level of the mosaic affecting the local background estimate used by SExtractor. For recovered objects that were not blended with bright neighbors the difference between their true and recovered fluxes was in line with the expected statistical error.

\subsection{Comparison between the Main and Verification Surveys}

Fluxes of objects in the part of the main survey that overlapped with the verification strip were compared. This comparison allows a rough estimate of the color-dependent flat field error, as 

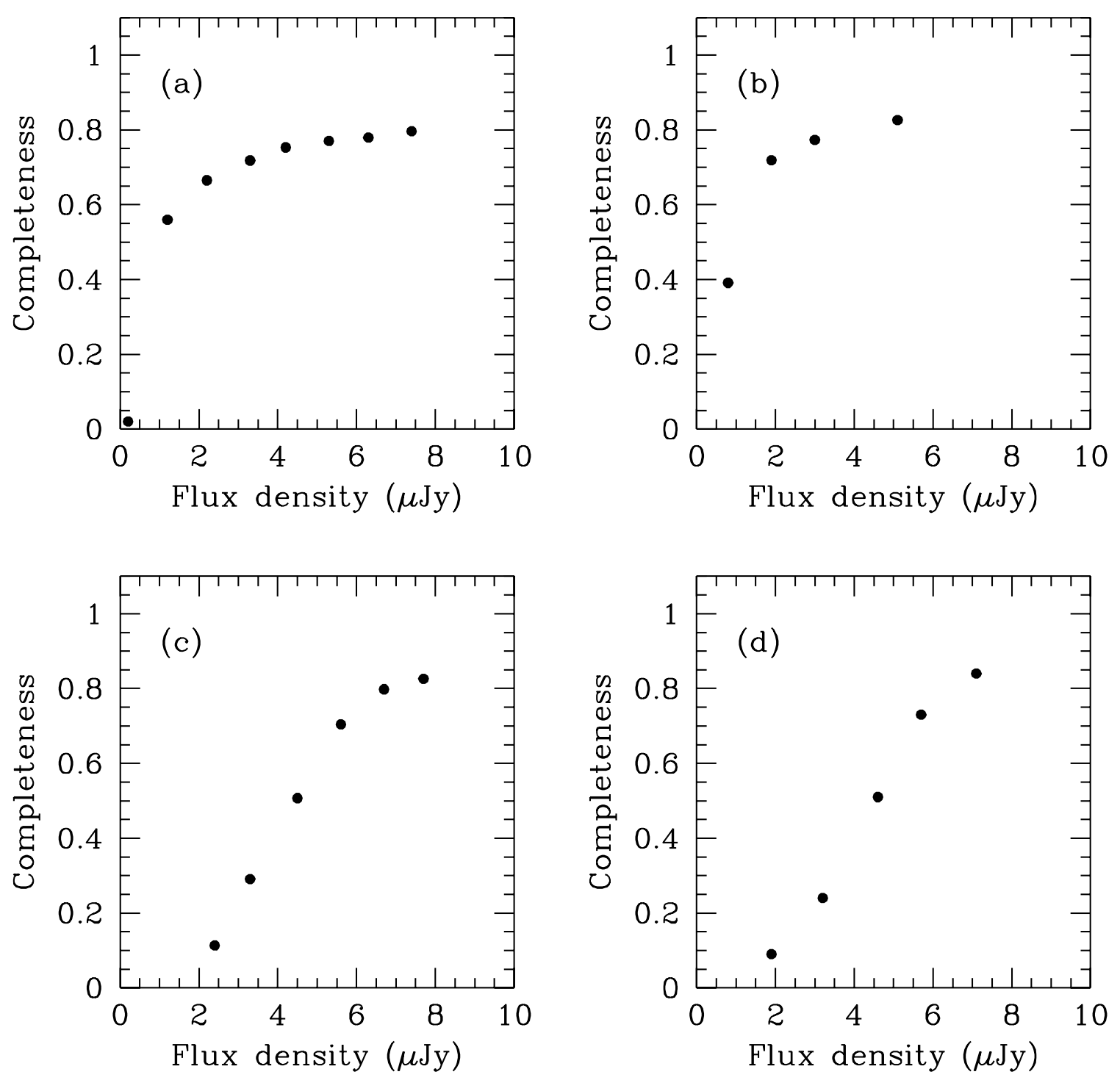

FIG. 4.- Results of completeness tests on the ELAIS-N1 field. The panels are for $(a)$ channel 1, $(b)$ channel 2, $(c)$ channel 3, and $(d)$ channel 4.

the main and verification surveys were taken with different grids, and of errors induced through overzealous cosmic-ray rejection in the shallower coverage main field data.

The results of this test showed that the scatter between the main field and verification strip fluxes is about $10 \%$ in all four bands for high signal-to-noise detections, roughly as expected from the known sources of systematic error. To get a true estimate of the error would require a more random superposition of the main and verification survey grids, thus this estimate (which corresponds to an $\approx 7 \%$ uncertainty if the main and verification regions are affected similarly) is strictly a lower limit.

The main field fluxes in all bands average a little lower than those in the verification survey, by about $2 \%$. This is probably due to a combination of factors. At the faint end, the fluxes are measured in small apertures in the shallower survey, frequently missing flux. At the bright end, the lower coverage of the main survey will lead to more frequent misidentification of the brightest object pixels as cosmic rays, and the consequent exclusion of the brightest object pixels from the final mosaics.

\subsection{Colors of 2MASS Stars}

We performed a further check on the photometry by studying the colors of field 2MASS stars in the XFLS main field, follow- ing Eisenhardt et al. (2004). We placed a color cut of $J-K<0.3$ on the 2MASS stars, corresponding approximately to the color of a F7 dwarf (Bessell \& Brett 1988), and only considered stars with $K<14$ to ensure accurate 2MASS colors. Also, we excluded stars with $K<10$ from the quantitative comparison in channel 1 as these were saturated in the IRAC images. For channels 2, 3, and 4 the mean stellar color is close to zero, as expected. In channel 1, our initial calibration showed a significant offset from zero, similar to that noted by Eisenhardt et al. (2004). Part of this is due to the expected nonzero colors of the stars.

TABLE 6

Offsets and Dispersion of the Colors of Blue Stars BETWEEN 2MASS AND IRAC

\begin{tabular}{|c|c|c|c|}
\hline Color & $\begin{array}{c}\text { Expected } \\
\text { Mean Value }^{\mathrm{a}}\end{array}$ & $\begin{array}{c}\text { Offset from } \\
\text { Expected Mean }\end{array}$ & $\begin{array}{l}\text { Dispersion around } \\
\text { Expected Mean }\end{array}$ \\
\hline$K-\operatorname{ch} 1 \ldots \ldots \ldots \ldots \ldots \ldots$ & 0.04 & +0.03 & 0.07 \\
\hline$K-\operatorname{ch} 2 \ldots \ldots \ldots \ldots \ldots \ldots$ & 0.02 & +0.00 & 0.12 \\
\hline$K-\operatorname{ch} 3 \ldots \ldots \ldots \ldots \ldots \ldots$ & 0.00 & -0.02 & 0.11 \\
\hline$K-\operatorname{ch} 4 \ldots \ldots \ldots \ldots \ldots \ldots$ & 0.00 & +0.02 & 0.12 \\
\hline
\end{tabular}

${ }^{\text {a }}$ From Bahcall \& Brett (1988). 

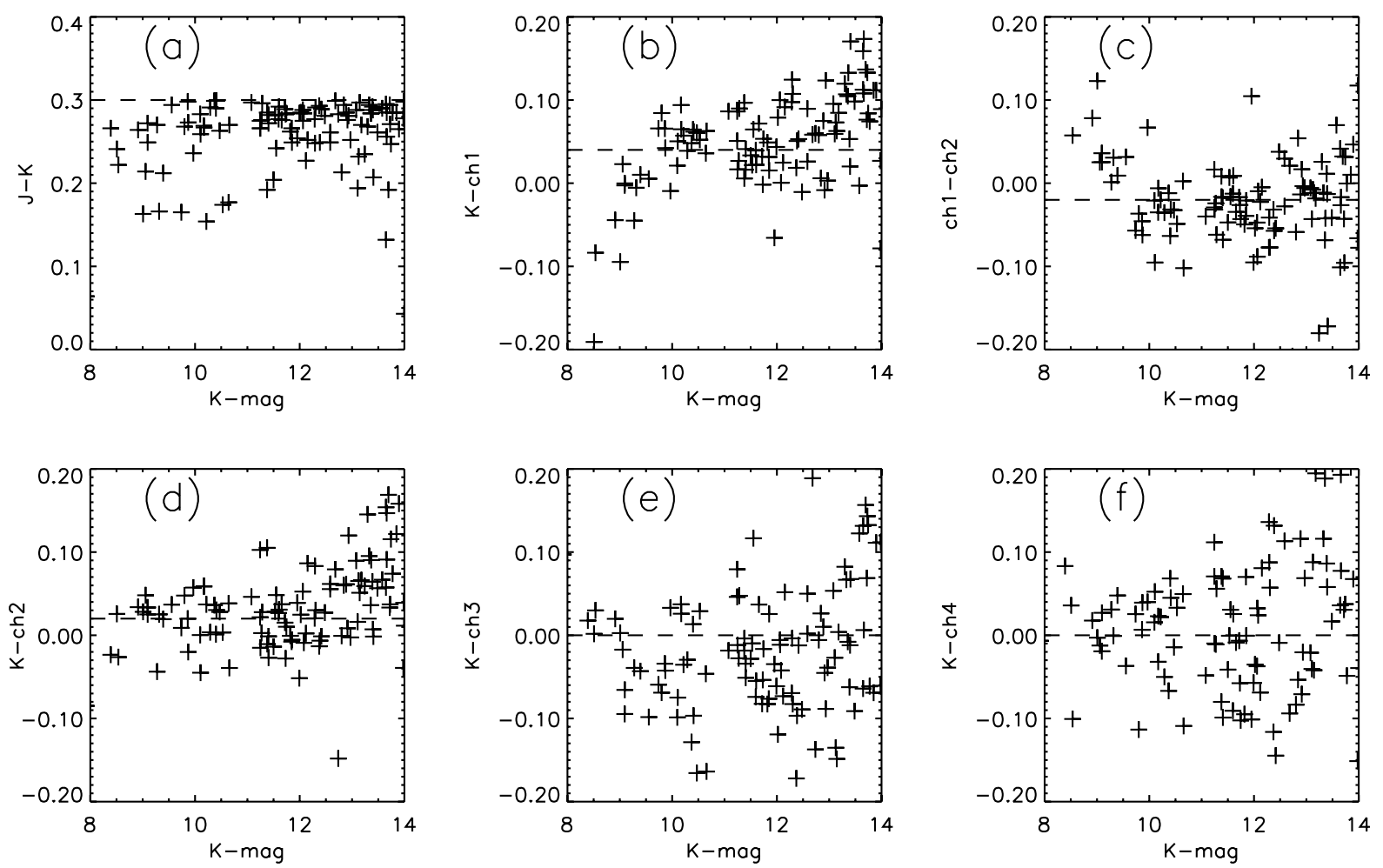

FIG. 5.-Photometry of blue stars in the XFLS field. (a) $J-K$ vs. $K$ color-magnitude diagram. The dashed line shows the $J-K=0.3$ color cut used to select the blue stars (corresponding to late-F and bluer). (b) $K-\operatorname{ch} 1$ vs. $K$. The dashed line at $K-\operatorname{ch} 1=0.04$ is the expected color of stars with $J-K=0.3$. (Stars brighter than $K \approx 10$ are saturated in the channel 1 image, producing the downturn at bright magnitudes.) $(c) \operatorname{ch} 1-\operatorname{ch} 2 \mathrm{vs} . K$. The dashed line at ch1 $-\operatorname{ch} 2=-0.02$ is the expected color of stars with $J-K=0.3$. (d) $K-\operatorname{ch} 2$ vs. $K$. The dashed line at $K-\operatorname{ch} 2=0.02$ is the expected color of stars with $J-K=0.3$, (e) $K-\operatorname{ch} 3$ vs. $K$, and $(f) K-\operatorname{ch} 4$ vs. $K$. In $(e)$ and $(f)$ the dashed line is at zero, the expected color in these pairs of bands.

Most of the stars are close to the red limit in $J-K$, which corresponds to a $K-L \approx K-\operatorname{ch} 1$ color of $\approx 0.04$ (see Table 6). However, a significant offset of $\sim 5 \%-8 \%$ still remained.

Based on these plots, we decided to change the flux conversion factor for channel 1 to the value determined from measuring the fluxes of the A-star calibrators only (T. Megeath 2004, private communication), $0.1085,4 \%$ different from that given in the S9.5/S10.5 BCD headers $(0.1125)$. This improves the channel 1 fluxes further, and the residual discrepancy is $\sim 3 \%$. This residual is comparable to that expected from the effects described above and to the known uncertainties in the channel 1 flat fields from IRAC campaigns 1 and 2. Figure 5 shows the final plots, and Table 6 the numerical results. Based on these, the flux density scale of channel 1 looks accurate to $\approx 3 \%$ both when compared to $K$-band (Fig. $5 b$ ) and to channel 2 (Fig. $5 c$ ). The apparent upswing in the mean color in the $K-\operatorname{ch} 2$ plot for $K>13$ (Fig. $5 d$ ) largely disappears when a redder color cut (which includes more objects) is used, so is probably not significant. Examination of Table 6 suggests that, indeed, our estimate of $10 \%$ systematic uncertainty in the flux densities of individual sources is reasonable and that the systematic uncertainty in the flux density scale is $\sim 2 \%-3 \%$. Unfortunately, there were too few stars in the verification region to allow us to perform the same test on the deeper data, but the comparison between the main and verification field flux densities showed only small systematic differences $(\S 7.2)$.

\section{SETTING THE FLUX DENSITY LIMITS}

As this survey is expected to be used primarily for statistical purposes, we have produced single-band catalogs cut at flux density levels at which the surveys are still $\approx 80 \%$ complete
(Table 7), and at which the simulations indicate that the mean fraction of flux recovered by SExtractor from point or slightly extended sources is $\gtrsim 90 \%$. This resulted in us setting limits in the main field catalog of 20,25,100, and $100 \mu \mathrm{Jy}$ in channels 1 , 2,3 , and 4 , respectively. The corresponding limits in the verification survey were $10,10,30$, and $30 \mu \mathrm{Jy}$, and those in the ELAIS-N1 field are $4,3,10$, and $10 \mu \mathrm{Jy}$. The survey limits scale approximately with the square root of the exposure time in channels $2-4$, but channel 1 is less deep than expected in the verification and ELAIS-N1 fields. Source confusion is certainly partly to blame, but in channel 2 similar source densities are reached in the deep surveys without a strong departure from the expected scaling. The most likely explanation is a combination of source confusion and the latent image problem, which was especially severe in the verification data in channel 1 and resulted in a varying background that was only partly corrected by the subtraction of a delta-dark. The flux density limits correspond to a signal-to-noise ratio of $\approx 5$ for a typical source at the limit of the surveys (Fig. 6), except in the deeper channel 1 data affected by variable background and confusion, where it is $\approx 7$.

We have compared our flux density limits with those of the Spitzer Performance Estimation Tool (PET, http://ssc.spitzer .caltech.edu/tools/pet.html). For the main field, we expect $5 \sigma$ sensitivities of $7.4,11,61$, and $60 \mu \mathrm{Jy}$ in channels $1-4$, respectively, compared to our survey limits of $20,25,100$, and $100 \mu \mathrm{Jy}$, respectively. In the verification survey, the PET gives $5 \sigma$ sensitivities of $1.8,3,20$, and $23 \mu \mathrm{Jy}$ compared to $10,10,30$, and $30 \mu \mathrm{Jy}$, and in ELAIS-N1, $0.33,0.7,4.6$, and $5.8 \mu \mathrm{Jy}$ compared with $4,3,10$, and $10 \mu \mathrm{Jy}$. There are three reasons why we do not achieve the PET sensitivities. First, the PET estimates are based on point-source fitting rather than aperture photometry. Point-source fitting has 7-13 noise pixels, depending on channel 
TABLE 7

Completeness and Confusion Statistics

\begin{tabular}{|c|c|c|c|c|c|}
\hline Survey & Channel & $\begin{array}{l}\text { Catalog Limit } \\
(\mu \mathrm{Jy})\end{array}$ & $\begin{array}{c}\text { Completeness at Limit } \\
(\%)\end{array}$ & Number of Sources & $\begin{array}{c}\text { Source Density } \\
\left(\mathrm{deg}^{-2}\right)\end{array}$ \\
\hline 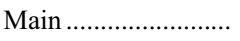 & 1 & 20 & 77 & 93689 & 24700 \\
\hline 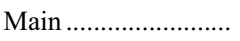 & 2 & 25 & 82 & 69915 & 18400 \\
\hline 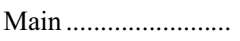 & 3 & 100 & 93 & 10792 & 2840 \\
\hline 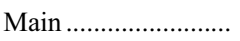 & 4 & 100 & 94 & 11891 & 3130 \\
\hline Verification ................... & 1 & 10 & 71 & 9339 & 37400 \\
\hline Verification .................... & 2 & 10 & 79 & 8652 & 34600 \\
\hline Verification .................. & 3 & 30 & 80 & 2904 & 11600 \\
\hline Verification ................... & 4 & 30 & 83 & 2517 & 10100 \\
\hline ELAIS-N1 ................... & 1 & 4 & 75 & 2113 & 103100 \\
\hline ELAIS-N1 ................... & 2 & 3 & 77 & 2381 & 116100 \\
\hline ELAIS-N1 .................... & 3 & 10 & 90 & 1374 & 67000 \\
\hline ELAIS-N1 .................... & 4 & 10 & 90 & 919 & 44800 \\
\hline
\end{tabular}
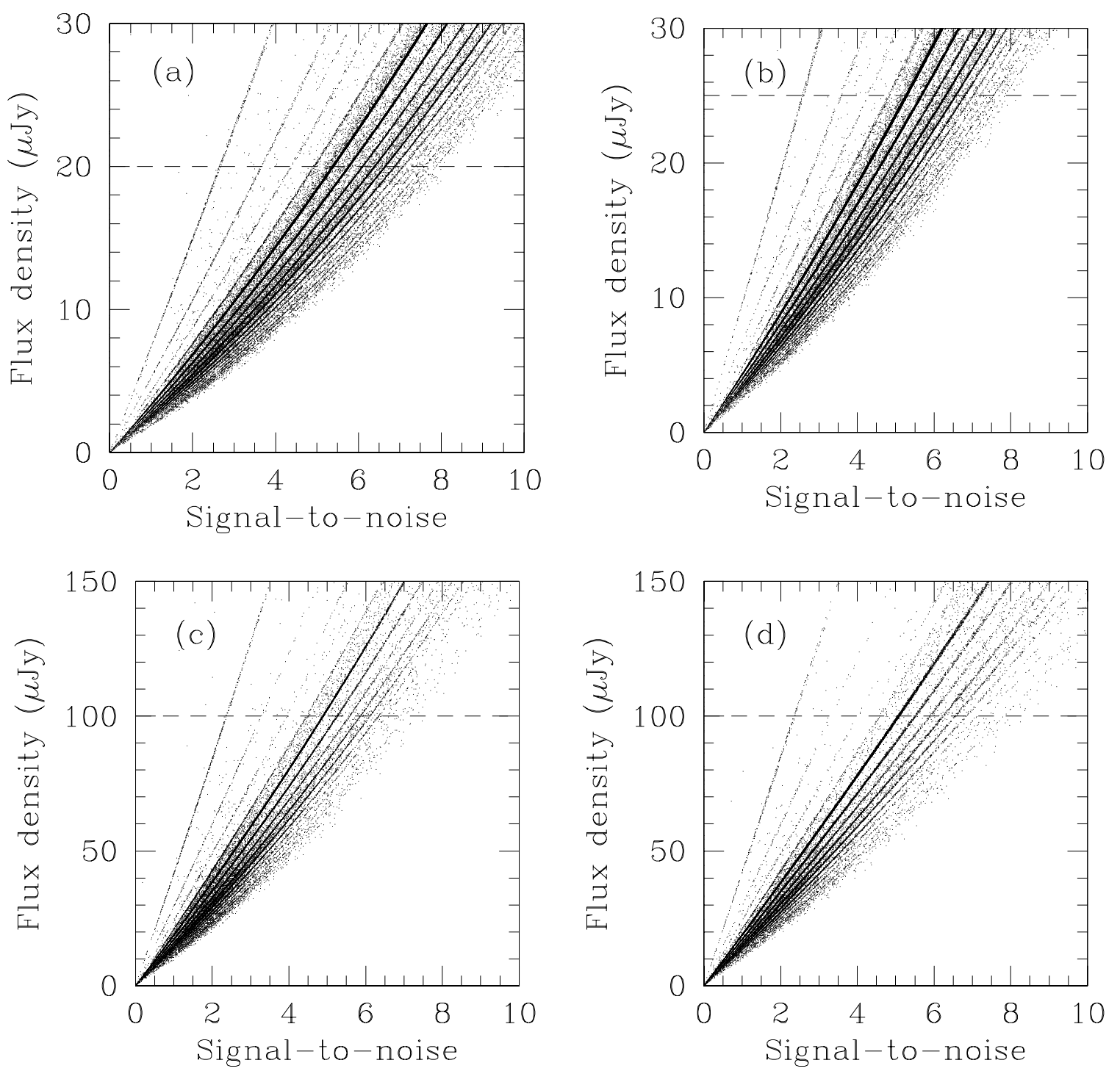

FIG. 6.-Flux density against signal-to-noise ratio in the "best" apertures for sources in the raw main field catalogs in each channel: $(a)$ channel $1,(b)$ channel 2 , (c) channel 3, and $(d)$ channel 4 . The dashed lines show the adopted flux density limits for the final catalogs in each band. The striations in the plots are the result of selecting the fluxes from a small set of fixed apertures. 
(see the Spitzer Observers' Manual), compared to 19 pixels in our smallest photometric aperture, which accounts for much of the difference in the main field survey, and in all surveys in channels 3 and 4. Second, the PET estimates take no account of source confusion. This is particularly important for channels 1 and 2 in the ELAIS-N1 survey. Third, the background variations in channel 1 discussed above limit our sensitivity in the deeper surveys in that channel.

\section{RELIABILITY}

Objects detected in the main field catalogs that overlapped the region covered by the verification data were compared with those made from the $30 \mathrm{~s}$ data in the verification strip, which goes much deeper. This allowed us to make an estimate of the reliability of the main field catalog at the catalog flux density limits given above. These tests showed that the catalogs are $\approx 99 \%$ reliable in all four bands, in the sense that the probability of an object in the main field catalog being detected in the deeper verification data was $\approx 99 \%$. Measuring the reliability of the verification and ELAIS-N1 catalogs is not possible using this technique, but as their limits are similar to the main field in terms of signal-to-noise ratio we expect these catalogs to be similarly reliable.

\section{DATA PRODUCTS}

\subsection{Mosaics}

A mosaic for each channel for the main and verification fields is available, along with coverage maps. All are made in the same fiducial frame, i.e., with the same pixel grid. This results in much of the verification mosaic being blank, but it greatly eases source comparison as the same pixels in every mosaic and in every channel correspond to the same sky position. The 12 and $30 \mathrm{~s}$ frames in the verification region are co-added according to inverse variance weights determined from the measured standard deviations in the BCDs. We opted to keep the main field data separate from the verification field data to maintain their statistical independence. The coverage maps show the numbers of frames added to produce the final mosaic. In the main field, one unit of coverage corresponds to one $12 \mathrm{~s}$ frame, in the verification field one unit is one $30 \mathrm{~s}$ frame, with the $12 \mathrm{~s}$ frames being 0.388 (the ratio of the exposure times in the 12 and $30 \mathrm{~s}$ data). Multiplying by the appropriate exposure time ( $10.4 \mathrm{~s}$ for the $12 \mathrm{~s}$ frames in the main field and $26.4 \mathrm{~s}$ for the $30 \mathrm{~s}$ frames in the verification field) will thus recover the exposure time for a given pixel.

\subsection{Catalogs}

\subsubsection{Single-Band Catalogs}

The single-band catalogs contain the following fields.

SrcID.-An integer identifier for the source.

$x, y$.-Pixel coordinates of the source in the mosaic.

R.A., decl.- Right ascension and declination of the source in the single-channel mosaic (J2000.0 coordinates).

Best flux.-Most reliable estimate of the flux density selected as described in $\S 4$.

Best aper.-The aperture for the "best" flux (in arcsec).

Err. best flux.-The uncertainty in the "best" flux.

$S: N$.- The signal-to-noise ratio in the "best" aperture.

6.00 apc.-Aperture-corrected flux density in a 6."00 diameter aperture.

9".26 apc.-Aperture-corrected flux density in a 9".26 diameter aperture.

14 ".86 apc.-Aperture-corrected flux density in a $14 . " 86$ diameter aperture.
TABLE 8

Numbers of Sources in Each Catalog as a Function of Band

\begin{tabular}{|c|c|c|c|}
\hline $\begin{array}{l}\text { Detection Flag Binary } \\
\text { (Decimal) }\end{array}$ & Main & Verification & ELAIS-N1 \\
\hline $0001(1) \ldots \ldots \ldots \ldots$ & 30758 & 2631 & 1271 \\
\hline 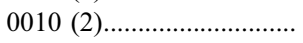 & 6984 & 2061 & 1622 \\
\hline 0011 (3) & 49534 & 3610 & 106 \\
\hline 0100 (4) & 822 & 139 & 655 \\
\hline 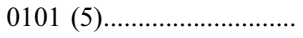 & 698 & 666 & 627 \\
\hline 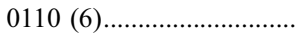 & 24 & 16 & 2 \\
\hline 0111 (7) ........................... & 2464 & 582 & 34 \\
\hline 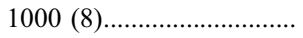 & 821 & 114 & 297 \\
\hline $1001(9) \ldots \ldots .$. & 61 & 8 & 1 \\
\hline 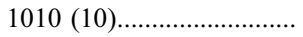 & 727 & 525 & 543 \\
\hline 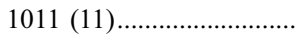 & 3510 & 368 & 25 \\
\hline 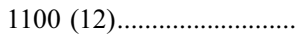 & 103 & 12 & 3 \\
\hline 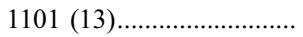 & 11 & 2 & 4 \\
\hline 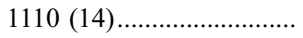 & 23 & 18 & 4 \\
\hline 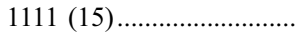 & 6653 & 1472 & 45 \\
\hline Total in catalog .................. & 103193 & 12224 & 5239 \\
\hline
\end{tabular}

24".4 apc.-Flux density in a 24".4 diameter aperture (no correction applied as this is the aperture the IRAC calibration stars are measured in).

Err 6".00, err 9".26, err 14".86, err 24".4.-Uncertainties in the aperture flux densities.

Area.-Area of the isophotal aperture (in pixels).

Ixx, Iyy, Ixy.- Second moments of the flux distribution.

Flag.- Sum of the flag bits; see Table 3.

Class.-Stellarity index from SExtractor (between 0 and 1, low values correspond to probable extended sources and high values to point-like sources).

Coverage.-Number of unit frames contributing to the mosaic at the source position (see $\S 10.1$ ).

All fluxes and errors are quoted in $\mu \mathrm{Jy}$. The errors include the statistical error and the $10 \%$ systematic uncertainty discussed in $\S 7$. The uncertainty in the overall flux density scale is not included, but we believe this to be small in any case, see $\S 7.3$ ). Table 7 summarizes the properties of the single-band catalogs.

\subsubsection{Four-Band Catalogs}

The four-band catalogs contain the following fields.

$x$, y.-Pixel coordinates of the source in the shortestwavelength mosaic in which it was detected.

R.A., decl.--Right ascension and declination of the source in the shortest-wavelength mosaic in which it was detected (J2000.0 coordinates).

ch1 id, ch2 id, ch3 id, ch4 id.-Identifiers in the four singleband catalogs (defaults to -1 for nondetections in a given band).

ch1 flux, err ch1.-Channel 1 flux density and uncertainty in the "best" aperture.

ch2 flux, ch2 err.-Channel 2 flux density and uncertainty. ch3 flux, ch3 err.-Channel 3 flux density and uncertainty. ch4 flux, ch4 err.-Channel 4 flux density and uncertainty. Aper.-Aperture used for flux density measurements. Flag.-See Table 4.

$d f l g$.- Sum of the detection flag bits, see discussion in $\S 4$.

Objects with no coverage in a given band have their flux densities in that band set to 99,999.0. Otherwise, nondetections have their fluxes set to the appropriate catalog limit, or a $3 \sigma$ limit, whichever is higher.

Table 8 shows the numbers of sources in each catalog as a function of detection flag. Of 93,689 channel 1 main field detections, 
$62,161(66 \%)$ are also detected in channel 2, $9826(10 \%)$ are detected in channel 3 , and 10,235 (11\%) are detected in channel 4.

\section{LESSONS LEARNED AND FUTURE WORK}

The observations for the XFLS were designed well before launch, when the knowledge of the properties of the instrument was rather limited. With the benefit of hindsight we would have changed a number of aspects of the design. The greater than expected scattered light problems, and the latent image problems in channel 1 both could have been mitigated by using a larger dither pattern (with arcminute-scale dithers) and/or half array offsets in the mapping strategy. On the other hand, one correct decision was to maintain a relatively high coverage factor ( 5 over the main field). This allowed reliable cosmic-ray rejection, and the extra redundancy allowed us to use the scattered light masks effectively.

Future reprocessings of the XFLS data should be able to improve the accuracy of the fluxes. For example, we may be able to use color-dependent flat fields to obtain more accurate fluxes by applying the flat field appropriate to the color of each object using an iterative technique. Also, we may be able to use improved source extraction techniques based on a development of the SSC point source extractor to optimally extract the typically slightly extended IRAC sources and properly quantify the confusion in the ELAIS-N1 channel 1 and 2 data. These improvements were considered to be beyond the scope of this work, where our purpose is to provide a reliable and fairly complete catalog on a reasonably short timescale.

\section{SUMMARY}

The IRAC data from the XFLS has been analyzed, and a set of catalogs produced that we believe to be $\approx 80 \%$ complete and $\approx 99 \%$ reliable. The final bandmerged catalogs contain 103,193 objects in the main field, 12,224 in the verification field, and 5239 in ELAIS-N1. Flux densities of high signal-to-noise objects are accurate to about $10 \%$, and the systematic uncertainty in the absolute flux density scale is $\sim 2 \%-3 \%$. Positional accuracy is $\approx 0$ ". 25 for high signal-to-noise objects and $\approx 1^{\prime \prime}$ at the flux density limits of the catalogs. We have successfully extracted sources at source densities as high as $100,000 \mathrm{deg}^{-2}$ in our deepest channel 1 and 2 data, though there are indications that we are approaching the confusion limit at these high source densities, in agreement with Fazio et al. (2004b). The mosaics and catalogs will be made available both through the Spitzer Science Archive and the NASA/IPAC Infrared Science Archive (IRSA).

We thank the other members of the IRAC instrument/ instrument support team, in particular Sean Carey, for helpful discussions, and Dan Stern and Lexi Moustakas for making their software available. We also thank I. Drozdovsky for supplying the mosaics of the ACS data in the XFLS field, and the anonymous referee for helpful comments. This work is based on observations made with the Spitzer Space Telescope, which is operated by the Jet Propulsion Laboratory, California Institute of Technology under NASA contract 1407. The Two Micron All Sky Survey (2MASS) is a joint project of the University of Massachusetts and the Infrared Processing and Analysis Center/ California Institute of Technology, funded by the National Aeronautics and Space Administration (NASA) and the National Science Foundation (NSF).
Bertin, E., \& Arnouts, S. 1996, A\&AS, 117, 393

Bessell, M. S., \& Brett, J. M. 1988, PASP, 100, 1134

Chary, R. et al. 2004, ApJS, 154, 80

Cutri, R. M. et al. 2003, Explanatory Supplement to the 2MASS All Sky Data Release (Pasadena: IPAC), http://www.ipac.caltech.edu/2mass/releases/allsky/ doc/explsup.html

Eisenhardt, P. R., et al. 2004, ApJS, 154, 48

\section{REFERENCES}

Fazio, G. G., et al. 2004a, ApJS, 154, 10

Fazio, G. G., et al. 2004b, ApJS, 154, 39

Frayer, D. T., et al. 2005, ApJS, submitted

Lacy, M., Gates, E. L., Ridgway, S. E.,. de Vries, W., Canalizo, G., Lloyd, J. P., \& Graham, J. R. 2002, AJ, 124, 3023

Masci, F. J., Makovoz, D., \& Moshir, M. 2004, PASP, 116, 842

Werner, M. W. et al. 2004, ApJS, 154, 1 